\title{
Spectrum of computed tomography manifestations of appendiceal neoplasms: acute appendicitis and beyond
}

\author{
Gita Yashwantrao Karande ${ }^{1}$, MBBS, FRCR, Wei Ming Chua ${ }^{1}$, MBBS, FRCR, Raphael Shih Zhu Yiin ${ }^{1}$, MBBS, FRCR, \\ Kang Min Wong ${ }^{1}$, MBBS, FRCR, Sandeep $\underline{\text { Hedgire }}^{2}$, MBBS, MD, Tien Jin $\underline{\operatorname{Tan}}^{1}$, MBBS, FRCR
}

\begin{abstract}
Appendiceal neoplasms are rare and often only discovered incidentally during surgery performed for acute appendicitis. Computed tomography (CT) has been demonstrated to be a reliable technique for accurately establishing the preoperative diagnosis of appendiceal neoplasms that manifest as acute appendicitis through the presence of certain imaging findings. Other manifestations of appendiceal neoplasms include appendiceal mass, mucocoele, localised abscess formation, ileus, increasing abdominal girth from pseudomyxoma peritonei, and intussusception. This pictorial essay illustrates varied CT findings of neoplasms of the appendix, with emphasis on the more commonly encountered manifestations of these tumours.
\end{abstract}

Keywords: appendiceal neoplasm, appendicitis, computed tomography, mucocoele, pseudomyxoma peritonei

\section{INTRODUCTION}

Appendiceal neoplasms are uncommon tumours that are mostly found incidentally during appendicectomies performed for appendicitis, accounting for $0.5 \%-1.0 \%$ of appendicectomy specimens. ${ }^{(1)}$ Given the frequent manifestation of appendiceal neoplasms as acute appendicitis, radiological imaging, particularly computed tomography (CT), is often performed for these patients and can lead to diagnosis. Other less common presentations may also be diagnosed on imaging, including appendiceal mass, mucocoele, intussusception, bowel obstruction, secondary genitourinary complications or complications such as perforation with abscess formation or ovarian lesion. ${ }^{(1)}$ It is important to detect appendiceal neoplasms early, as the prognosis is usually poor if they are found at an advanced stage. Early detection is also important because it may change the surgical approach (open vs. laparoscopic surgery) and the surgical procedure (appendicectomy vs. right hemicolectomy). ${ }^{(2)}$ In this pictorial essay, we aimed to highlight the common CT features of appendiceal neoplasms, other less frequent but important manifestations and potential imaging pitfalls.

\section{ACUTE APPENDICITIS}

The vermiform appendix is a blind-ended outpouching that typically arises from the posteromedial caecal wall about $2 \mathrm{~cm}$ below the ileocaecal valve. On imaging, the normal appendix measures $6 \mathrm{~mm}$ or less in maximal outer diameter (Fig. 1). ${ }^{(3)}$

Often considered as a non-essential vestigial organ, the appendix is better known for its pathological role in acute appendicitis, one of the most common abdominal surgical emergencies. Acute appendicitis occurs when there is obstruction of the appendiceal lumen, leading to fluid accumulation, luminal distension and inflammation before finally resulting in perforation. CT remains the imaging modality of choice in evaluating adult patients presenting with acute appendicitis because of its high diagnostic accuracy. ${ }^{(4)}$

On CT, acute appendicitis is diagnosed when there are signs of appendiceal thickening and inflammation. Appendiceal thickening is defined as the appendix having an outer diameter greater than $6 \mathrm{~mm}$. Inflammatory changes include appendiceal wall thickening measuring $3 \mathrm{~mm}$ or greater and mural hyperenhancement. Other supportive findings such as periappendiceal fat stranding, extraluminal fluid, phlegmon, appendicolith and lymphadenopathy may be observed. ${ }^{(4)}$

\section{APPENDICEAL NEOPLASMS}

Appendiceal neoplasms are important entities to consider in patients presenting with acute appendicitis. About 30\%$50 \%$ of appendiceal neoplasms initially manifest as acute appendicitis. (1) Primary appendiceal neoplasms have a diverse histology comprising of epithelial tumours (mucinous and colonic subtypes), neuroendocrine tumours (NETs), goblet cell carcinoids, lymphoma, mesenchymal tumours and sarcomas. ${ }^{(3)}$ NETs are the commonest, followed by epithelial neoplasms. In comparison, the other neoplasms are much rarer. ${ }^{(1,3,5)}$ The histology subtype and extent of disease at the time of diagnosis help to predict survival. For example, the presence of a signet-ring cell formation confers the worst prognosis with an average survival rate of six months, while a low-grade mucinous neoplasm gives a median overall survival of eight years. ${ }^{(5)}$ Prompt diagnosis of appendiceal neoplasms is therefore essential.

\section{CT FEATURES OF COMMON APPENDICEAL NEOPLASMS \\ Neuroendocrine tumours}

NETs are usually less than $1 \mathrm{~cm}$ in diameter and are often established incidentally in postoperative appendicectomy specimens. Most are located at the tip of the appendix and are

1Department of Radiology, Changi General Hospital, Singapore, ${ }^{2}$ Department of Radiology, Harvard Medical School - Massachusetts General Hospital, Boston, Massachusetts, USA Correspondence: Dr Gita Yashwantrao Karande, Senior Resident, Department of Radiology, Changi General Hospital, 2 Simei Street3, Singapore 529889. gitaykarande@gmail.com 

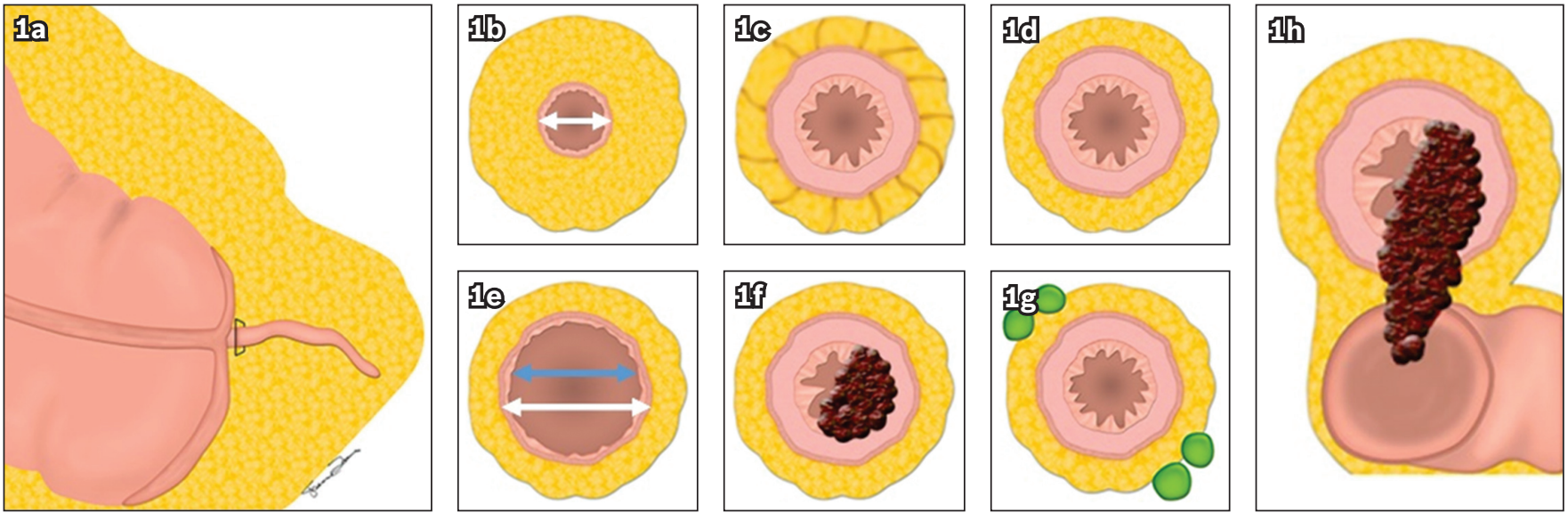

Fig. 1 (a) Gross anatomical graphical illustration of the caecum and appendix with cross sections through the appendix shows key radiological findings in (b) a normal appendix with a diameter $<6 \mathrm{~mm}$; and (c) non-tumoral appendicitis with appendix diameter $>6 \mathrm{~mm}$, mural thickening and periappendiceal fat stranding. $(\mathrm{d}-\mathrm{h})$ Illustrations of an appendix with an appendiceal neoplasm show $(\mathrm{d})$ a paucity of periappendiceal fat stranding; (e) outer diameter $>15 \mathrm{~mm}$ (white double arrow) or luminal diameter > $13 \mathrm{~mm}$ (blue double arrow); (f) intraluminal soft-tissue nodularity; (g) periappendiceal lymphadenopathy; and $(\mathrm{h})$ infiltration of adjacent structures.
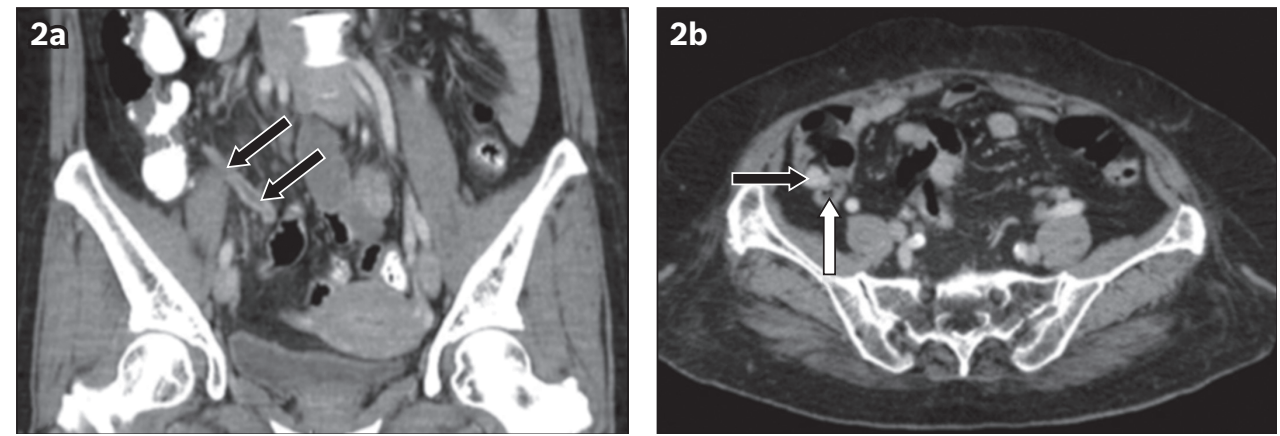

Fig. 2 (a) Contrast-enhanced coronal CT image of a 48-year-old woman with a proven carcinoid tumour of the appendix shows appendiceal mural thickening and hyperenhancement (arrows) with no significant periappendiceal fat stranding. (b) Contrast-enhanced axial CT image of an 80-year-old woman with an appendiceal carcinoid tumour shows a dense nodular lesion mimicking an appendicolith (black arrow) at the tip of the appendix (white arrow).
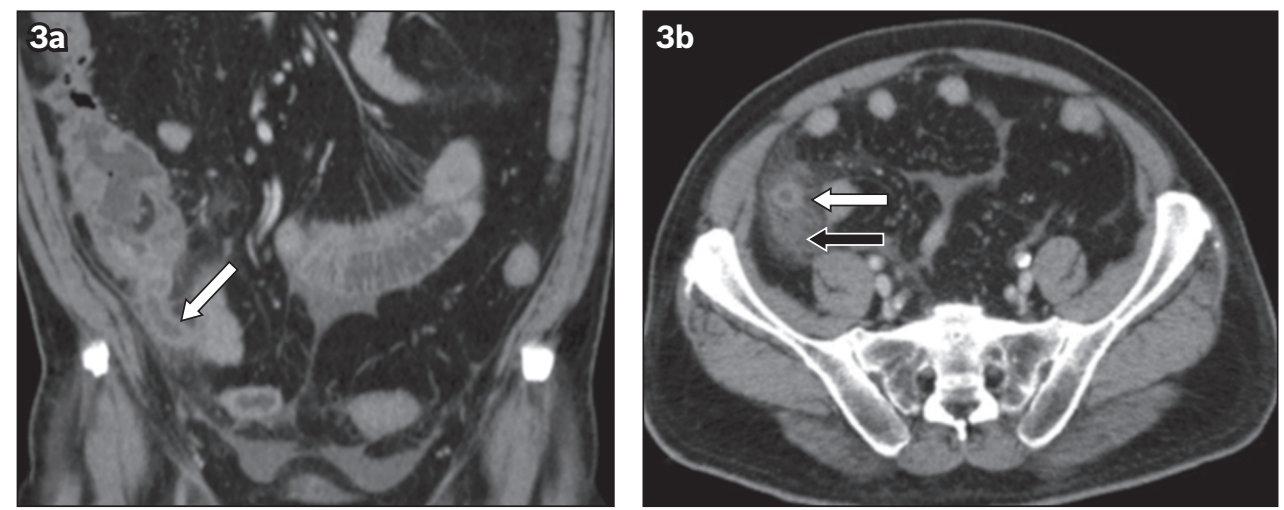

Fig. 3 A 69-year-old man presented with right iliac fossa pain. Contrast-enhanced (a) coronal and (b) axial CT images show diffuse mural thickening (white arrows in $\mathrm{a} \& \mathrm{~b}$ ) and periappendiceal fat stranding (black arrow) without cystic dilatation or discrete soft-tissue mass. The serendipitous diagnosis of invasive moderately differentiated adenocarcinoma of the appendix was only made following appendicectomy and histologic examination.

difficult to identify on CT due to their small size. NETs frequently show imaging findings of acute appendicitis and may mimic appendicoliths (Fig. 2). In metastatic disease, classic features reminiscent of small bowel carcinoids are seen, such as an irregular mesenteric mass with calcification and a surrounding desmoplastic reaction. NETs have little metastatic potential, occurring primarily in tumours greater than $2 \mathrm{~cm}$ in size and usually involving the regional lymph nodes. ${ }^{(1,3)}$

\section{Non-mucinous (colonic-type) epithelial tumours}

Non-mucinous epithelial tumours consist of adenomas, adenocarcinomas and polyps. These may obstruct the appendiceal lumen and often result in acute appendicitis (Fig. 3). Adenocarcinoma is the most common subtype that is detected on imaging. Apart from the imaging features of acute appendicitis, a soft-tissue mass or soft-tissue infiltration of the appendix may be seen (Fig. 4). Other imaging findings include direct invasion of 

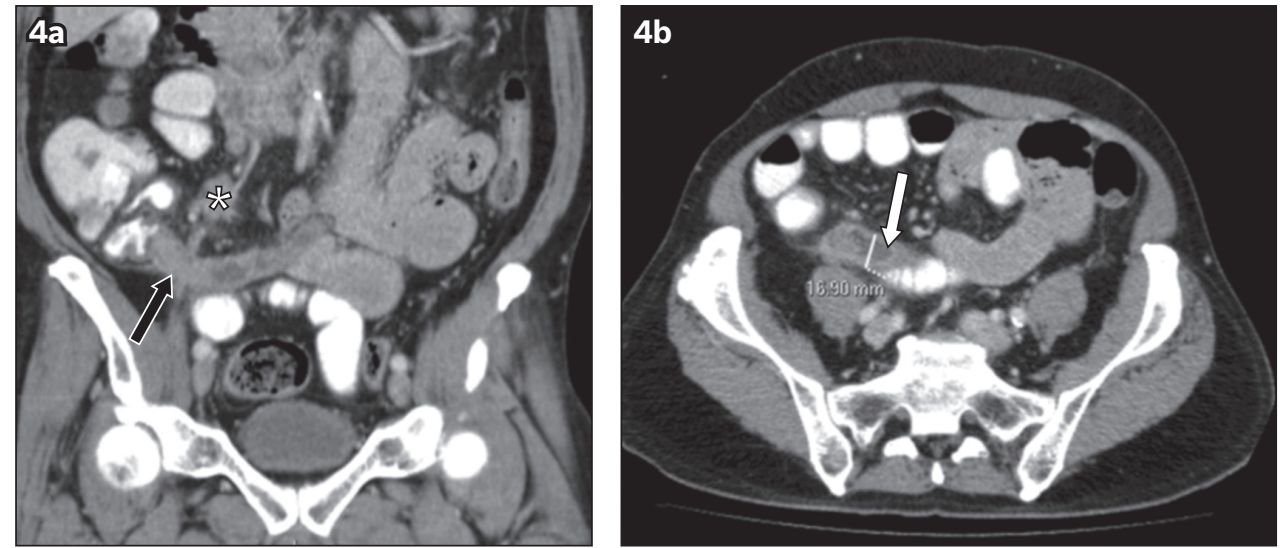

Fig. 4 A 66-year-old man presented with right iliac fossa pain. Contrast-enhanced (a) coronal and (b) axial CT images show cystic dilatation of the appendiceal tip (white arrow) and diffuse soft-tissue infiltration at the appendiceal base (black arrow) with minimal periappendiceal fat stranding. An enlarged ileocolic lymph node is also present (asterisk). This is suspicious for tumoral acute appendicitis. The diagnosis of a moderately differentiated appendiceal adenocarcinoma was established following surgery and histologic examination.
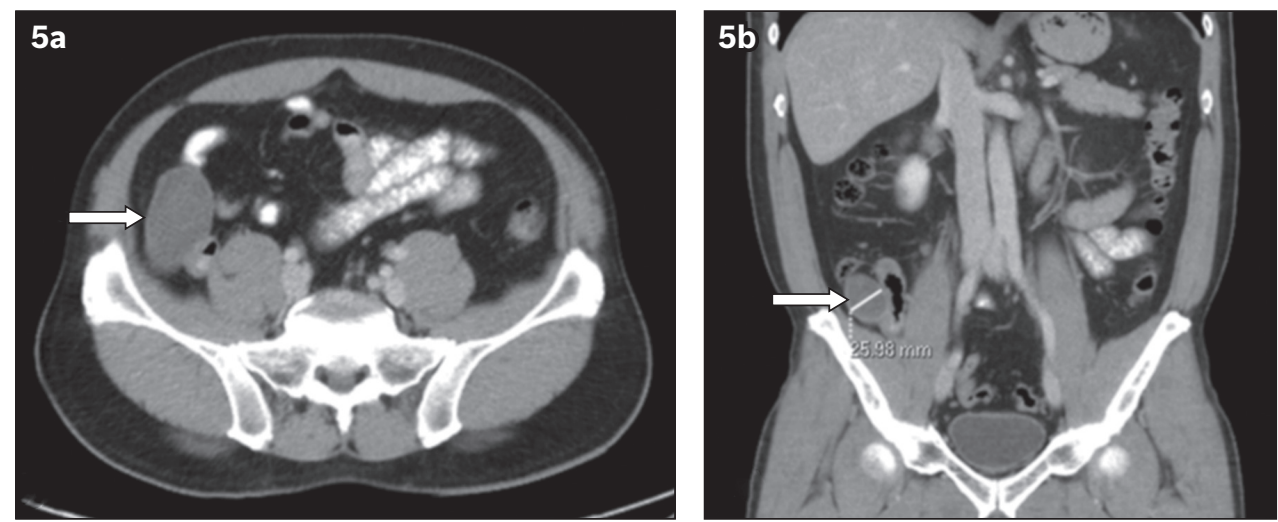

Fig. 5 A 50-year-old man presented with right lower abdominal pain. Contrast-enhanced (a) axial and (b) coronal CT images show a well-encapsulated tubular cystic structure in the right iliac fossa (arrows in a \& b) with minimal surrounding fat stranding. There is communication with the caecum (not shown), raising suspicion for an appendiceal mucocoele. Histology confirmed appendiceal mucocoele secondary to low-grade appendiceal mucinous neoplasm.

the adjacent organs, regional and distant lymphadenopathy, as well as metastasis. The rate of distant metastasis at presentation is reported to be $37 \%$, the most common sites being the peritoneum, lymph nodes (Fig. 4), ovaries, liver and lungs. ${ }^{(1,3)}$

\section{Mucinous epithelial tumours}

The majority of appendiceal epithelial tumours are mucinous. Mucinous neoplasms are generally classified as adenomas, lowgrade appendiceal mucinous neoplasms, high-grade appendiceal mucinous neoplasms and mucinous adenocarcinomas (MACAs). MACAs demonstrate infiltrative invasion and are further classified as well, moderately or poorly differentiated. ${ }^{(3)}$

Most mucinous neoplasms present as mucocoeles and are less likely to manifest as appendicitis than other appendiceal neoplasms. This is because mucocoeles result from chronic luminal obstruction without significant inflammation and generally require superinfection to develop into acute appendicitis. Other manifestations comprise of palpable mass, increasing abdominal girth from pseudomyxoma peritonei (PMP), intussusception, torsion and right ureteral obstruction. ${ }^{(1)}$

\section{Mucocoeles}

Appendiceal mucocoele is defined as dilatation of the appendiceal lumen due to abnormal mucus accumulation. ${ }^{(1,3)} \mathrm{CT}$ can be used to diagnose an appendiceal mucocoele with high accuracy. ${ }^{(1)}$ The typical appearance is a round or tubular well-encapsulated cystic mass with little periappendiceal inflammation (Fig. 5) and sometimes demonstrating calcification (Fig. 6). The punctate or curvilinear mural calcification is related to a dystrophic response to chronic inflammation and may result in a porcelain appendix. ${ }^{(6)}$ In female patients, it should be differentiated from a cystic ovarian neoplasm, tubo-ovarian abscess or hydrosalpinx, which can have a similar appearance. Identification of a normal right ovary can help exclude these ovarian pathologies. Other differential diagnoses are enteric duplication cyst and mesenteric cyst. ${ }^{(1)}$

Mucocoeles can be caused by neoplastic and non-neoplastic conditions. An attempt should be made to differentiate between these two broad groups, as they have differing clinical courses. ${ }^{(7)}$ Generally, neoplastic conditions should be considered when mucocoeles are larger than $2 \mathrm{~cm}$, while non-neoplastic causes rarely result in dilatation larger than $2 \mathrm{~cm} .{ }^{(8)}$ Reported CT features suggestive of a malignant mucocoele are wall irregularity, internal soft-tissue attenuation and enhancing nodularity (Figs. 7 \& 8). ${ }^{(7)}$

Acute appendicitis with and without a coexisting mucocoele can be clinically indistinguishable. It is vital to identify the presence of a mucocoele, as the surgical approach needs to be altered to avoid rupture and consequently PMP, for which satisfactory treatment is usually difficult to achieve. 

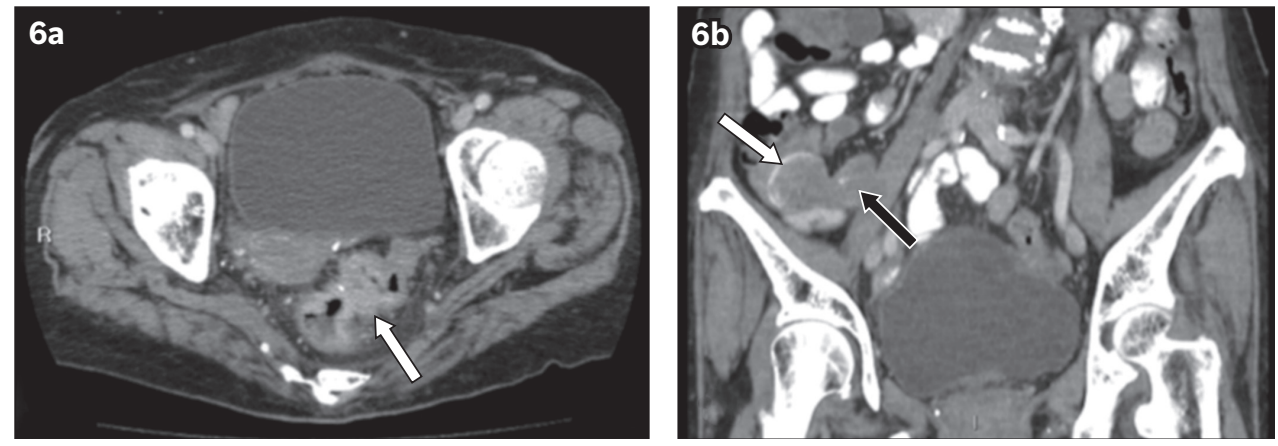

Fig. 6 An 86-year-old woman presented with generalised abdominal pain. (a) Contrast-enhanced axial CT image shows irregular circumferential mural thickening and enhancement in the rectosigmoid (arrow) suggestive of malignancy. (b) Coronal CT image shows tubular dilatation of the appendix with low attenuation contents (black arrow) and curvilinear mural calcification (white arrow). Histology confirmed synchronous rectosigmoid adenocarcinoma and appendiceal mucocoele secondary to low-grade appendiceal mucinous neoplasm.
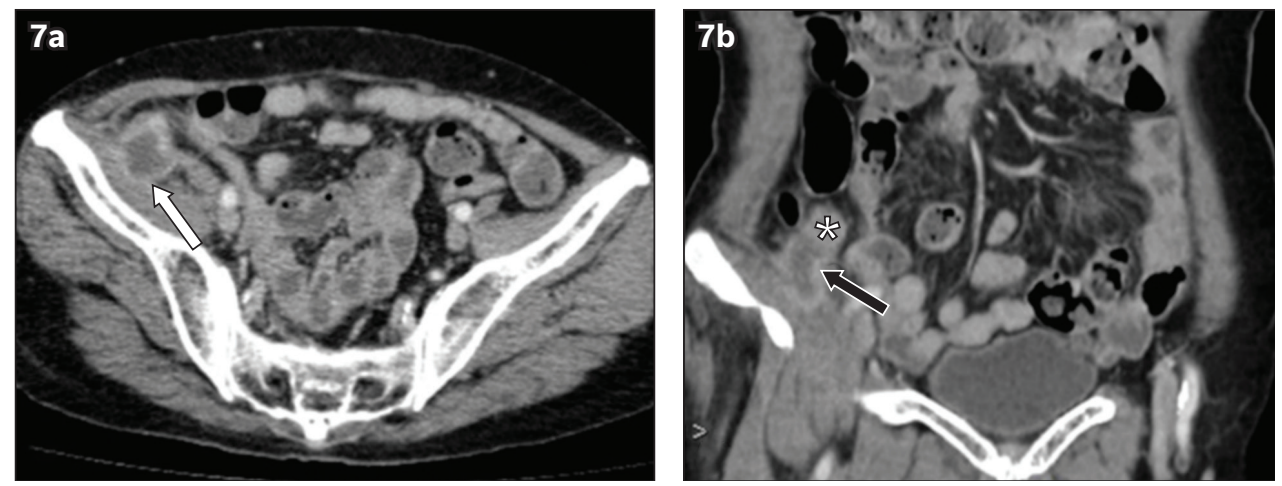

Fig. 7 An 87-year-old woman presented with infiltrative mucinous adenocarcinoma of the appendix. Contrast-enhanced (a) axial and (b) coronal CT images show cystic appendiceal dilatation (black arrow) with nodular mural thickening and enhancement (asterisk). There is a paucity of adjacent inflammatory changes. The appendix was adherent to the adjacent iliacus muscle (white arrow). The tumour was found to be perforated at the time of surgery.
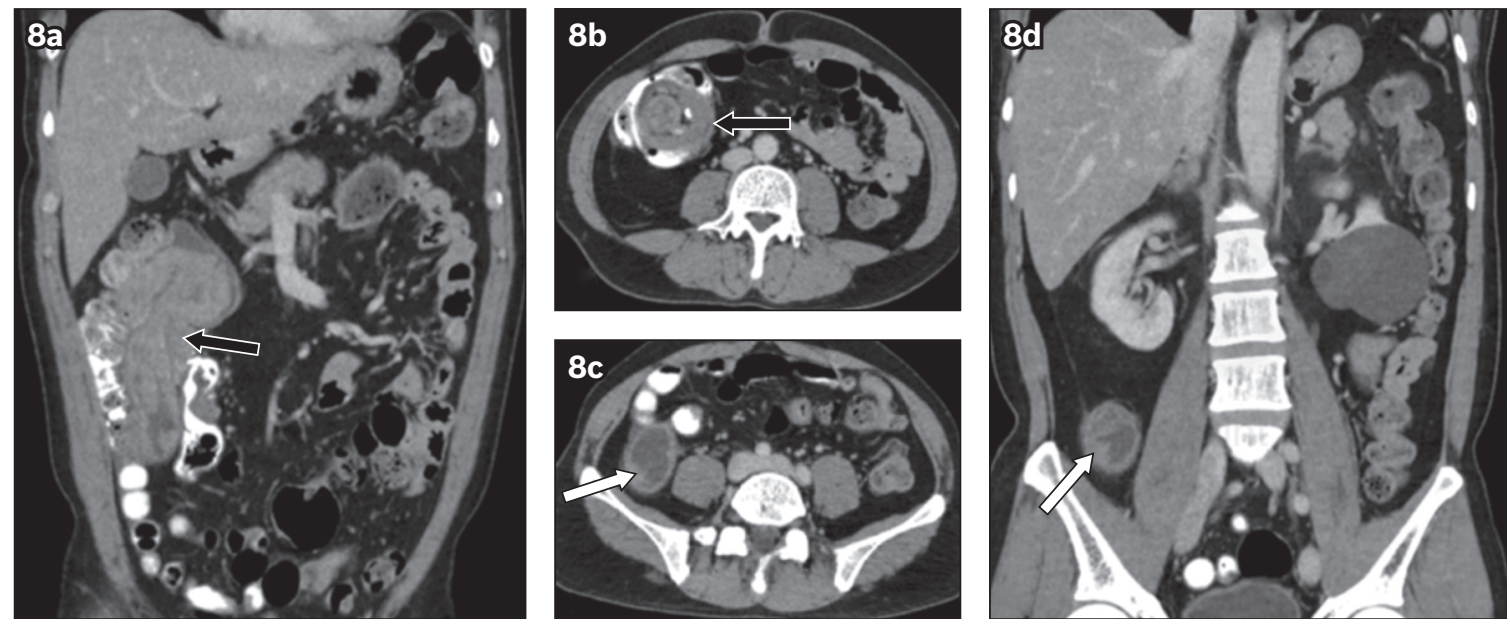

Fig. 8 A 50-year-old man presented with right iliac fossa pain. Contrast-enhanced (a) coronal and (b) axial CT images show appendico-colic intussusception (arrows in a \& b) with a 'target-like' appearance in (b). (c) Axial and (d) coronal CT images show enhancing mural soft tissue in the dilated appendix (arrows in c \& d), raising suspicion for underlying malignancy. Histopathological examination revealed a low-grade appendiceal mucinous neoplasm in association with tubulovillous adenoma.

CT features suggestive of a coexisting mucocoele encompass cystic dilatation of the appendix, mural calcification, luminal diameter greater than $1.3 \mathrm{~cm}$, or an outer diameter greater than $1.5 \mathrm{~cm}$. In particular, when the maximal luminal diameter of the appendix is greater than $1.3 \mathrm{~cm}$, the sensitivity and specificity for diagnosing a mucocoele are $71.4 \%$ and $94.6 \%$, respectively. ${ }^{(9)}$

\section{Pseudomyxoma peritonei}

PMP is a clinical syndrome defined as intraperitoneal accumulation of mucus due to mucinous neoplasia and is characterised by redistribution phenomenon. It includes mucinous ascites, peritoneal implants, omental caking and ovarian involvement. PMP can occur in both low- and high-grade lesions and may be due to spontaneous or iatrogenic perforation of the appendix. ${ }^{(3,7)}$ 

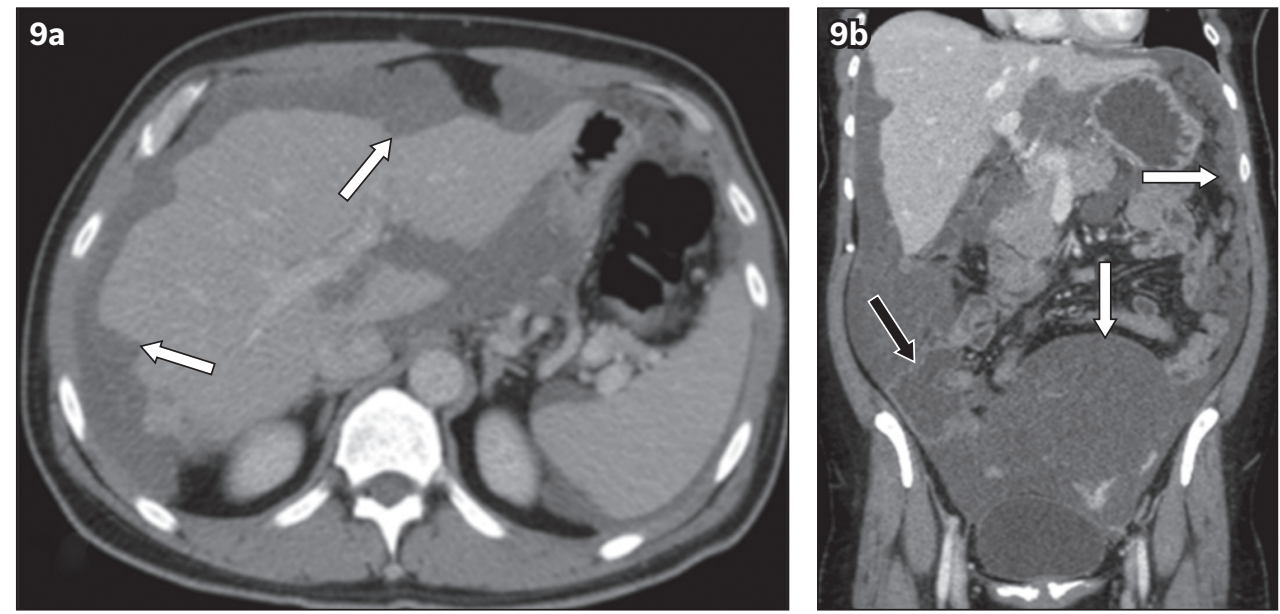

Fig. 9 A 53-year-old man presented with pseudomyxoma peritonei manifesting as increasing abdominal girth that resulted from a ruptured mucinous adenocarcinoma of the appendix. Contrast-enhanced CT (a) axial and (b) coronal CT images show widespread low-attenuation intraperitoneal locules that scallop the hepatic contour (arrows in a) causing a mass effect on the bowel loops (black arrow in b) as well as the mesentery (white arrows in b).
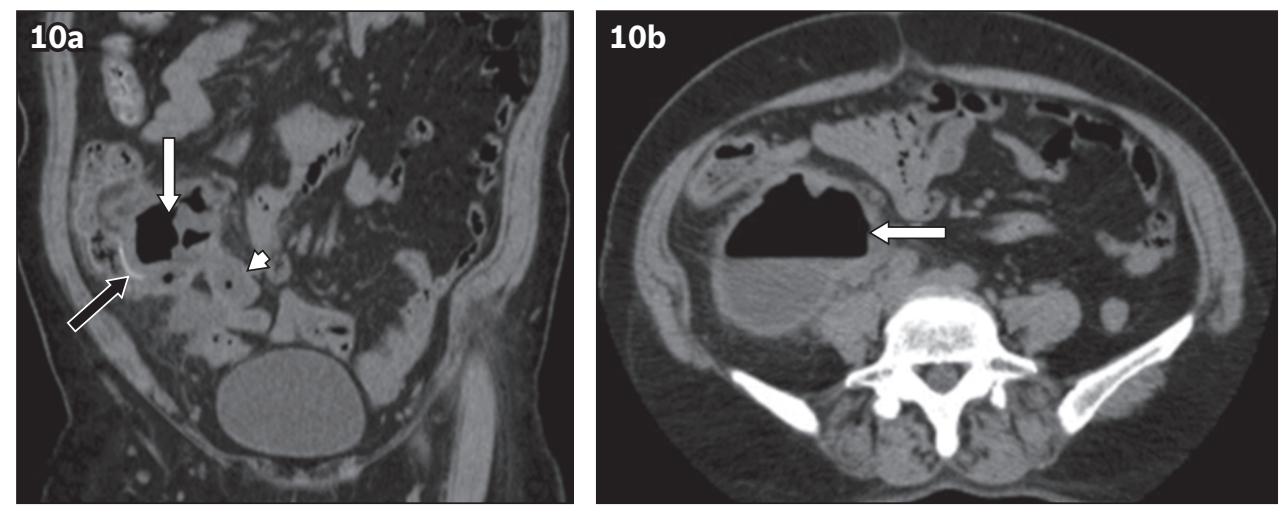

Fig. 10 A 62-year-old woman presented with right iliac fossa pain. Unenhanced (a) coronal and (b) axial CT images show an abscess adjacent to the base of the appendix (white arrows in a \& b) with dilatation of the tip of the appendix (arrowhead). Thin mural calcification (black arrow) is also noted. Histologic examination following surgery showed low-grade mucinous neoplasm of the appendix.

It most commonly arises from appendiceal neoplasms, but other associated conditions include ovarian, colon, rectum, stomach, pancreas and urachal tumours. ${ }^{(3)}$ Imaging findings are related to foreign body peritonitis with fibrotic response caused by spillage of mucus into the peritoneum. ${ }^{(6)}$

The diagnostic sign on CT is low-attenuation ascites with visceral scalloping due to implants (Fig. 9). ${ }^{(6)}$ Implants are seen on serosal surfaces and nidi in cavities reached by the usual routes of intraperitoneal flow dynamics, and they may show rim-like calcification. ${ }^{(6)}$ Frequent sites of implants are the pelvis, paracolic gutters, omentum and liver capsule. ${ }^{(3)}$ Adhesions and intestinal obstruction are the most frequent complications. ${ }^{(6)}$ In cases of concomitant mucinous tumours of the appendix and ovaries, there is often controversy about the origin of the tumour cells. However, recent evidence points to a likely appendiceal origin with secondary involvement of the peritoneum and ovaries. $^{(3)}$

\section{OTHER MANIFESTATIONS AND PITFALLS IN IMAGING \\ Perforation and abscess formation}

In general, it is difficult to differentiate perforated nontumoral appendicitis from perforated appendicitis secondary to an underlying appendiceal neoplasm. ${ }^{(5,9)}$ In such patients, histopathology and follow-up imaging play an important role in its distinction (Figs. 10-13).

\section{Intussusception}

Intussusception in adults is uncommon, comprising about $5 \%$ of all intussusceptions and $1 \%$ of all cases of bowel obstruction. The majority of cases are caused by a neoplastic lead point (approximately 65\%). The commonest site is the small bowel, followed by the large bowel. ${ }^{(10)}$ By comparison, intussusception of the appendix is even rarer, accounting for $4 \%$ of all adult intussusceptions and $0.01 \%$ of appendicectomy specimens. The few reported cases in the available literature describe lead points consisting of an endometriotic deposit, appendiceal mucocoele, primary malignancy or metastasis. ${ }^{(11)}$ Intussusceptions with a neoplastic lead point are usually longer (more than $3.6 \mathrm{~cm}$ ) and have larger diameters (greater than $3.4 \mathrm{~cm}$ ) than the non-neoplastic ones, while the degree of the dilation of the proximal small bowel is greater with a neoplastic lead point. ${ }^{(10)}$

The pathognomonic CT appearance is the bowel-withinbowel configuration, which may contain fat or mesenteric vessels (Fig. 8). ${ }^{(10)}$ Subhepatic caecum and an appendix 

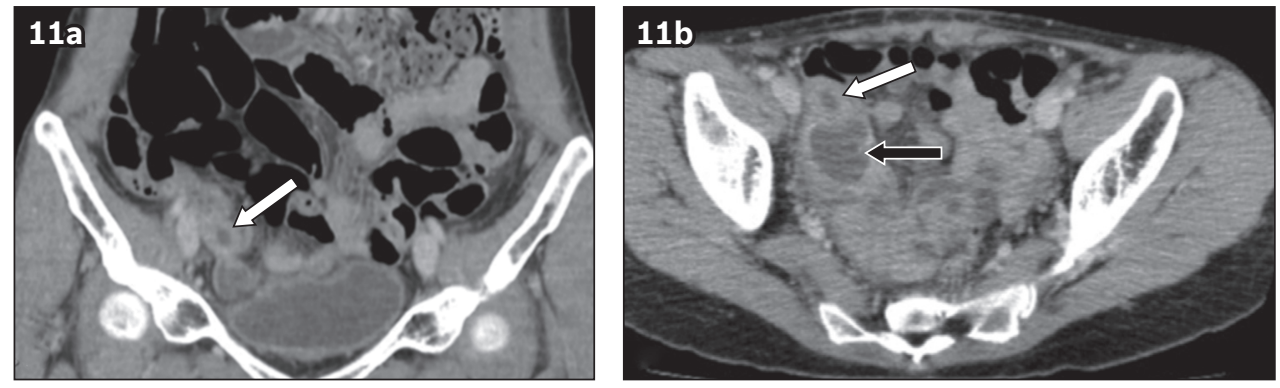

Fig. 11 A 45-year-old woman presented with right iliac fossa pain. Contrast-enhanced (a) coronal and (b) axial CT images show dilatation of the appendix with thickened enhancing walls (white arrows in a \& b). There is a contained perforation with abscess formation at the tip of the appendix (black arrow in b). The diagnosis of low-grade appendiceal mucinous neoplasm was made after histopathological examination.
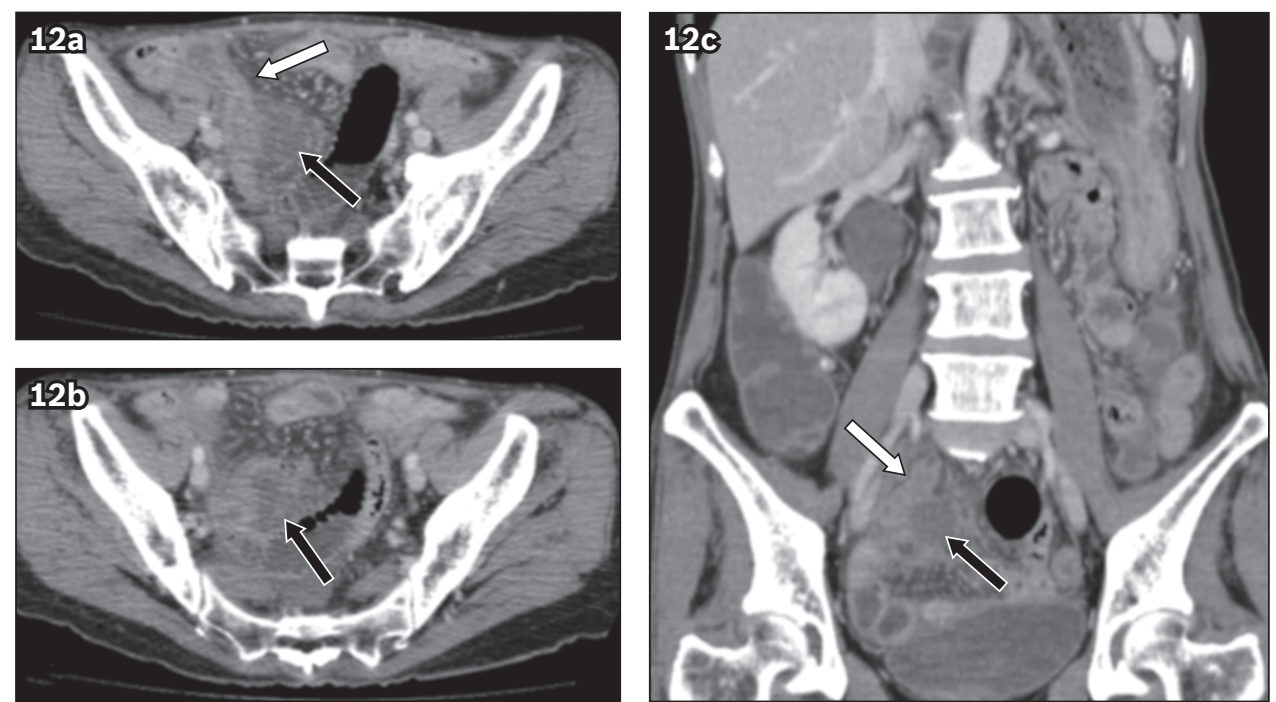

Fig. 12 Contrast-enhanced ( $\mathrm{a} \& \mathrm{~b}$ ) axial and (c) coronal CT images of a 56-year-old woman show appendiceal mural thickening and enhancement (white arrows in $\mathrm{a} \& \mathrm{c}$ ) with perforation and abscess formation (black arrows in $\mathrm{a}-\mathrm{c}$ ). A low-grade appendiceal mucinous neoplasm was found on histopathological examination.
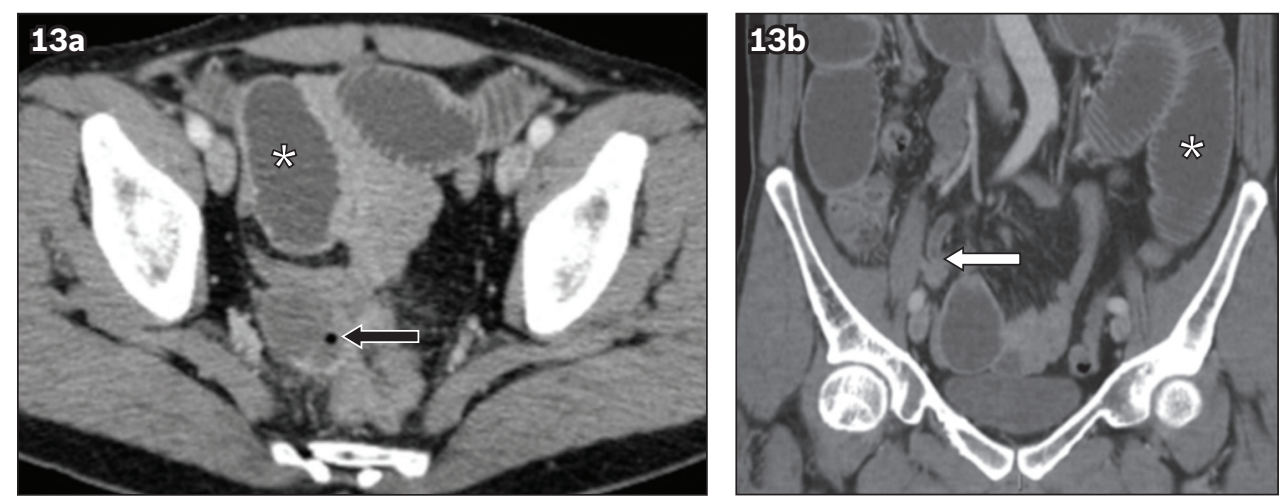

Fig. 13 A 46-year-old man with abdominal pain. Contrast-enhanced (a) axial and (b) coronal CT images show appendiceal mural thickening and enhancement with perforation at the tip (white arrow) and a resultant rim-enhancing abscess containing a gas pocket (black arrow). Dilatation of small bowel loops is suggestive of small bowel ileus (asterisk). Perforated mucinous adenocarcinoma was diagnosed postoperatively following histologic examination.
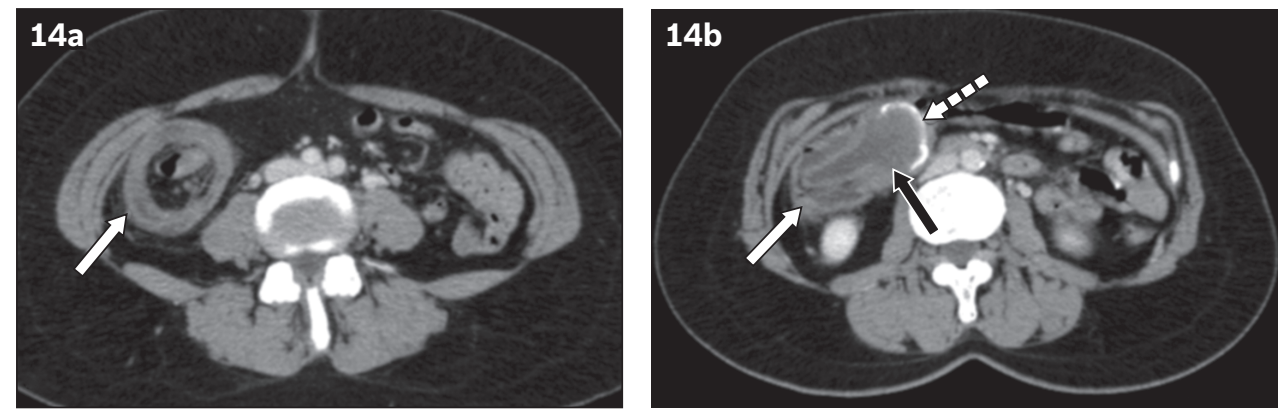

Fig. 14 A 57-year-old woman presented with right iliac fossa pain. Contrast-enhanced axial CT images show ileo-appendicocolic intussusception (white arrows in a \& b) with (a) 'target-like' and (b) 'cup and ball' appearance in a patient with mucocoele of the appendix (black arrow in b). The dilated appendix with mural calcification (dashed arrow in b) was in keeping with a low-grade appendiceal mucinous neoplasm on histopathological examination. 

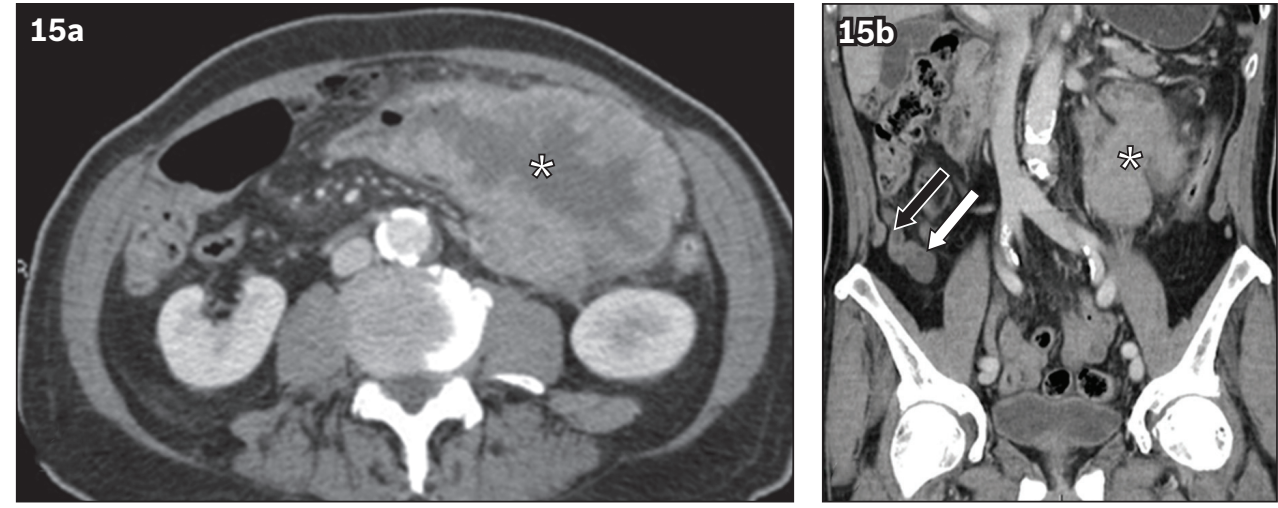

Fig. 15 A 79-year-old man had synchronous non-mucinous, colonic-type adenocarcinoma of the appendix and transverse colon. (a) Contrast-enhanced axial CT image shows irregular mural thickening and enhancement in the transverse colon (asterisks in a \& b). (b) Coronal CT image shows soft tissue (white arrow) at the tip of the mildly dilated appendix (black arrow), in keeping with a synchronous tumour.
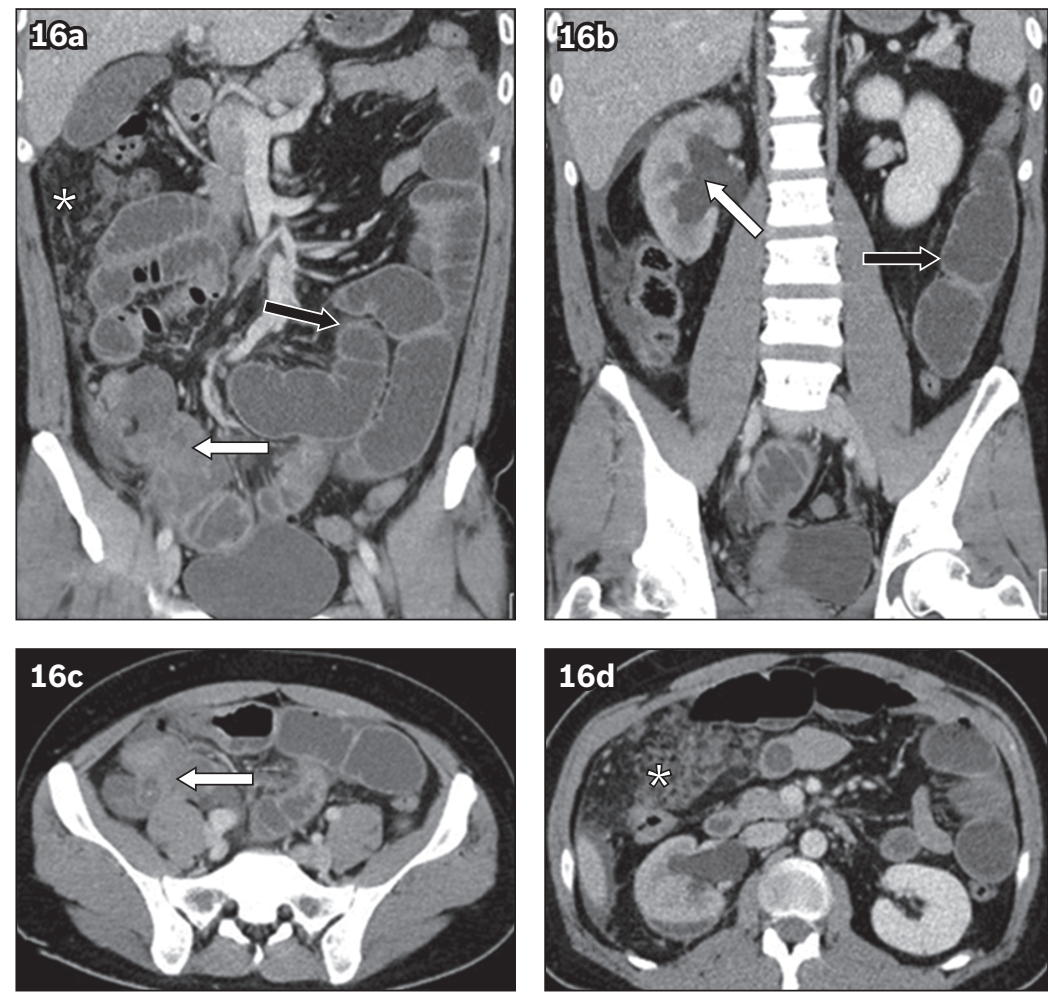

Fig. 16 A 35-year-old woman with abdominal pain. Contrast-enhanced ( $\mathrm{a} \& \mathrm{~b}$ ) coronal and ( $\mathrm{c} \& \mathrm{~d}$ ) axial CT images show a heterogeneous mass in the right lower abdomen (white arrows in a \& c). There is omental thickening and nodularity (asterisks in a \& d) as well as mechanical small bowel obstruction (black arrows in a \& b). The mass involves the right ureter with resultant hydronephrosis (white arrow in b). Histopathology revealed a multifocal mucinous adenocarcinoma involving the appendix, mesentery and gallbladder (not seen on imaging).

surrounded by a rim of mesenteric structures displaying a 'target-like' appearance can be observed (Figs. 8 \& 14). ${ }^{(12)}$ When an appendiceal mucocoele acts as a lead point, a 'cup and ball' pattern may be seen (Fig. 14). ${ }^{(11,12)}$ The absence of a normal appendix in such cases should alert the radiologist to the presence of an appendiceal mucocoele rather than other causes such as an enteric duplication cyst. Coronal reformations may aid in the diagnosis by demonstrating that the appendiceal lesion acts as a lead point, invaginating and drawing the terminal ileum and the ileocolic mesentery into the caecum and the ascending colon. CT also aids in the diagnosis of complications such as perforation and gives valuable information about the presence or absence of metastasis and lymphadenopathy.

\section{Synchronous appendiceal and colorectal neoplasm}

It has been reported that a quarter of patients with appendiceal cancer are found to have a synchronous or metachronous lesion elsewhere in the large intestine, as the appendix is embryologically derived from the large intestine and has a similar mucosal pattern (Figs. $6 \& 15$ ). ${ }^{(13)}$ The incidental finding of a synchronous appendiceal or colorectal neoplasm on preoperative imaging would understandably alter the surgical approach.

\section{Small bowel obstruction}

Mechanical obstructions caused by appendiceal neoplasms are rare. ${ }^{(14)}$ An appendiceal neoplasm is often missed when it presents as small bowel obstruction, as the clinical features of small bowel obstruction may predominate the clinical picture. 

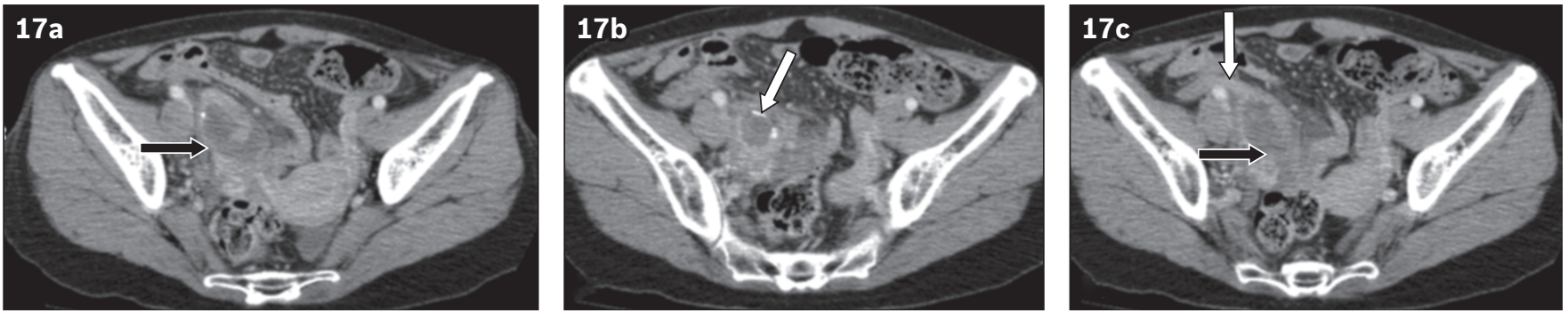

Fig. 17 A 56-year-old woman presented with right iliac fossa pain. (a-c) Contrast-enhanced axial CT images show an abscess in the right adnexal region (black arrows in a \& c) that is suspicious for a tubo-ovarian abscess. The possibility of a perforated appendiceal mucocoele was also raised in view of the close proximity of this lesion to the appendiceal tip (white arrow in c) and presence of curvilinear calcification (arrow in b). Histopathologic examination revealed a mucinous cystadenoma of the appendix.
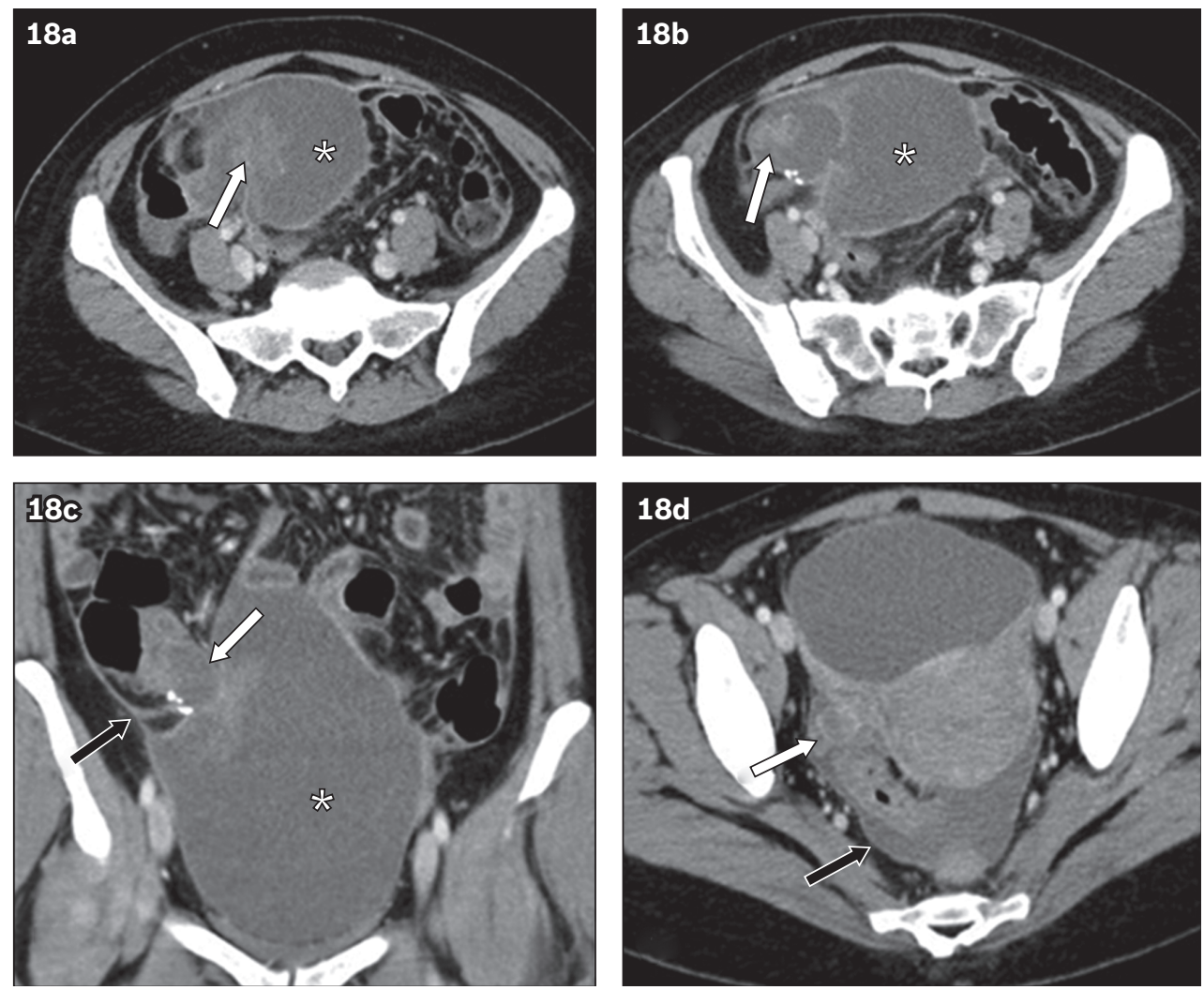

Fig. 18 A 47-year-old woman presented with pain in the right iliac fossa. (a \& b) Contrast-enhanced axial CT images show a predominantly cystic mass (asterisks) in the right lower quadrant with an enhancing eccentric solid component (arrows in a \& b). (c) Coronal and (d) axial CT images show peritoneal thickening and enhancement (black arrows in $\mathrm{c} \& \mathrm{~d}$ ). A preoperative diagnosis of a complex right ovarian mass with peritoneal deposits was made. The diagnosis of a low-grade appendiceal neoplasm with pseudomyxoma peritonei was established following surgery. In retrospect, (c) coronal CT image shows a short dilated tubular structure suggestive of the appendix (white arrow in c) leading into this cystic mass (asterisk in c). There is presence of calcification as well. (d) Axial CT image shows normal right ovary with corpus luteum (white arrow in d).

CT helps in diagnosing the location, cause and degree of obstruction. If the zone of transition is in the right iliac fossa, it is important to consider an appendiceal pathology in the list of differential diagnoses for the cause of small bowel obstruction (Fig. 16). ${ }^{(14)}$ The operating surgeon should be warned of an appendiceal neoplasm acting as the cause of obstruction, as this will alter the surgical approach and management, such as the extent of bowel resection.

\section{OVARIAN PATHOLOGY}

An appendiceal neoplasm may mimic an ovarian pathology due to overlapping imaging features (Fig. 17). Both appendiceal mucinous and ovarian tumours can present as cystic lesions with eccentric enhancing solid components. Hence, they may be indistinguishable even by advanced imaging methods and remain undiagnosed until the time of surgery (Fig. 18). ${ }^{(8)}$

\section{Hydroureteronephrosis}

Appendiceal neoplasm causing hydroureteronephrosis is an uncommon but known complication. A right lower quadrant mass with resultant hydroureteronephrosis should prompt the reporting radiologist to consider an underlying appendiceal neoplasm (Fig. 16). ${ }^{(15)}$

\section{CONCLUSION}

In all patients being evaluated for lower abdominal pathologies, the appendix should be looked at and carefully assessed for the presence of suspicious features (Fig. 1). Knowledge of the 
spectrum of possible CT manifestations of appendiceal neoplasms, which may present concomitantly with or masquerade as acute appendicitis, is crucial for both surgeons and reporting radiologists to accurately establish a preoperative diagnosis of these uncommon tumours. Preoperative detection of these neoplasms is essential because it may change the surgical approach and obviate the need for additional surgery.

\section{ACKNOWLEDGEMENT}

We would like to thank Dr Ahmad M Aljefri, Consultant Radiologist, King Fahd Medical City, Medical Imaging Administration, Riyadh, Kingdom of Saudi Arabia, for contributing the CT images for Fig. 9.

\section{REFERENCES}

1. Pickhardt PJ, Levy AD, Rohrmann CA Jr, Kende Al. Primary neoplasms of the appendix: radiologic spectrum of disease with pathologic correlation. Radiographics 2003; 23:645-62.

2. Pickhardt PJ, Levy AD, Rohrmann CA, Kende AI. Primary neoplasms of the appendix manifesting as acute appendicitis: CT findings with pathologic comparison. Radiology 2002; 224:775-81.

3. Leonards LM, Pahwa A, Patel MK, et al. Neoplasms of the appendix: pictorial review with clinical and pathologic correlation. Radiographics 2017; 37:1059-83.

4. Pinto LN, Pereira JM, Cunha R, Pinto P, Sirlin C. CT evaluation of appendicitis and its complications: imaging techniques and key diagnostic findings. AJR Am
J Roentgenol 2005; 185:406-17.

5. Teixeira FJR Jr, Couto Netto SDD, Akaishi EH, et al. Acute appendicitis, inflammatory appendiceal mass and the risk of a hidden malignant tumor: a systematic review of the literature. World J Emerg Surg 2017; 12:12.

6. Madwed D, Mindelzun R, Jeffrey RB Jr. Mucocele of the appendix: imaging findings. AJR Am J Roentgenol 1992; 159:69-72.

7. Wang $\mathrm{H}$, Chen YQ, Wei R, et al. Appendiceal mucocele: a diagnostic dilemma in differentiating malignant from benign lesions with CT. AJR Am J Roentgenol 2013; 201:W590-5.

8. Akman L, Hursitoglu BS, Hortu I, et al. Large mucinous neoplasm of the appendix mimicking adnexal mass in a postmenopausal woman. Int J Surg Case Rep 2014; 5:1265-7.

9. Bennett GL, Tanpitukpongse TP, Macari M, Cho KC, Babb JS. CT diagnosis of mucocele of the appendix in patients with acute appendicitis. AJR Am J Roentgenol 2009; 192:W103-10.

10. Warshauer DM, Lee JK. Adult intussusception detected at CT or MR imaging: clinical-imaging correlation. Radiology 1999; 212:853-60.

11. Coulier B, Pestieau S, Hamels J, Lefebvre Y. US and CT diagnosis of complete cecocolic intussusception caused by an appendiceal mucocele. Eur Radiol 2002; 12:324-8.

12. Laalim SA, Toughai I, Benjelloun B, Majdoub KH, Mazaz K. Appendiceal intussusception to the cecum caused by mucocele of the appendix: laparoscopic approach. Int J Surg Case Rep 2012; 3:445-7.

13. Lohsiriwat $\mathrm{V}$, Vongjirad A, Lohsiriwat D. Incidence of synchronous appendiceal neoplasm in patients with colorectal cancer and its clinical significance. World J Surg Oncol 2009; 7:51.

14. Komo T, Kohashi T, Hihara J, et al. Intestinal obstruction caused by low-grade appendiceal mucinous neoplasm: a case report and review of the literature. Int J Surg Case Rep 2018; 51:37-40.

15. Ahmed K, Hoque R, El-Tawil S, Khan MS, George ML. Adenocarcinoma of the appendix presenting as bilateral ureteric obstruction. World J Surg Oncol 2008; $6: 23$. 


\title{
SINGAPORE MEDICAL COUNCIL CATEGORY 3B CME PROGRAMME
} (Code SMJ 201904B)

\author{
Question 1. Regarding appendiceal neoplasms: \\ (a) Acute appendicitis is a frequent manifestation. \\ (b) Magnetic resonance imaging is the modality of choice for imaging. \\ (c) Early detection plays an important role in surgical management. \\ (d) They usually have a good prognosis even if discovered at an advanced stage.
}

\section{Question 2. Regarding primary appendiceal neoplasms:}
(a) Epithelial neoplasms are the commonest.
(b) Low-grade appendiceal neoplasms have a good prognosis.
(c) Only histology helps to predict survival.
(d) More than $75 \%$ of patients present with acute appendicitis.

Question 3. Regarding neuroendocrine tumours of the appendix:
(a) They are mostly located in the appendiceal body.
(b) They are usually less than $1 \mathrm{~cm}$ in size.
(c) They have little metastatic potential.
(d) They usually metastasise to the lymph nodes.

Question 4. Regarding imaging findings of mucinous epithelial neoplasms:
(a) Mucinous epithelial neoplasms commonly manifest as acute appendicitis.
(b) Non-neoplastic mucocoeles are generally less than $2 \mathrm{~cm}$ in diameter.
(c) Enhancing nodularity is suggestive of a neoplastic mucocoele.
(d) Pseudomyxoma peritonei most commonly results from rupture of ovarian neoplasms.

Question 5. Tumoral appendicitis is suspected on imaging when:

(a) Appendiceal diameter is more than $15 \mathrm{~mm}$.

(b) There is a paucity of fat stranding.

(c) Periappendiceal lymphadenopathy is seen.

(d) There is infiltration of adjacent structures.

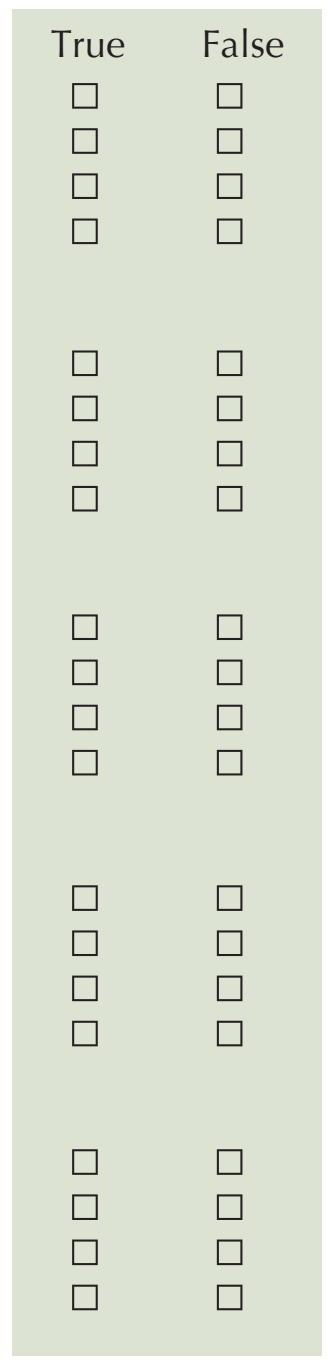

\section{Doctor's particulars:}

Name in full:

Specialty:

MCR no.:

Email:

\section{SUBMISSION INSTRUCTIONS}

Visit the SMJ website: http://www.smj.org.sg/current-issue and select the appropriate quiz. You will be redirected to the SMA login page.

For SMA member: (1) Log in with your username and password (if you do not know your password, please click on 'Forgot your password?'). (2) Select your answers for each quiz and click 'Submit'.

For non-SMA member: (1) Create an SMJ CME account, or log in with your SMJ CME username and password (for returning users). (2) Make payment of SGD 21.40 (inclusive of $7 \%$ GST) via PayPal to access this month's quizzes. (3) Select your answers for each quiz and click 'Submit'.

RESULTS:

(1) Answers will be published online in the SMJ June 2019 issue. (2) The MCR numbers of successful candidates will be posted online at the SMJ website by 10 June 2019. (3) Passing mark is $60 \%$. No mark will be deducted for incorrect answers. (4) The SMJ editorial office will submit the list of successful candidates to the Singapore Medical Council. (5) One CME point is awarded for successful candidates. (6) SMC credits CME points according to the month of publication of the CME article (i.e. points awarded for a quiz published in the December 2017 issue will be credited for the month of December 2017, even if the deadline is in January 2018).

Deadline for submission (April 2019 SMJ 3B CME programme): 12 noon, 3 June 2019. 